\title{
Entorno territorial y expectativas del agua para Bogotá
}

pags $160-171$

Grupo de Investigación: Desarrollo y equidad

Línea de investigación: Economía Social y Distribución del Ingreso y la Riqueza

Gustavo Sandoval Betancour•

\section{RESUMEN}

En el artículo se analizan aspectos relacionados con la seguridad de disponibilidad de agua en Bogotá, considerado las amenazas que inciden en este aspecto. Como un recurso para amortiguar los efectos de tales amenazas, se analiza como estrategia la difusión del aprovechamiento del agua lluvia con el fin de complementar los servicios de la Empresa de Acueducto de Bogotá y obtener las ventajas económicas y sociales que se obtienen con el mismo.

Palabras clave: Agua lluvia - Gobernanza del agua - Reservorios de agua

$$
\text { Clasificación JEL: H83 - Q54 - G5 }
$$

\section{ABSTRACT}

Safety aspects of water availability in Bogotá are analyzed, considering the threats affecting this aspect. As a resource to cushion the effects of such threats, analyze the strategy of spreading the use of rain water in order to supplement the services of the Bogota Water Company and get the economic and social advantages obtained therewith.

Keywords: Rainwater - Governance of water - Water reservoirs

Classification JEL: H83 - Q54 - G5

\footnotetext{
- Estudiante coinvestigador del programa de Ingeniería mecánica. Fundación Universidad de América. juan.caldas@estudiMg.Ec. Universidad Nacional de Colombia. Docente investigador, director del grupo de investigación Desarrollo y Equidad, Universidad de América, Bogotá Colombia. gustavo.sandoval@investigadores.uamerica.edu.co
} 
LIINEA DE INVESTIGACIÓN: ECONOMIA SOCIAL Y DISTRIBUCIÓN DEL INGRESO Y LA RIQUEZA

\section{INTRODUCCIÓN}

La crisis del agua amenaza a la humanidad, con mayor fuerza en algunos países que en otros. Sin embargo Colombia disfruta de una situación privilegiada en cuanto a disponibilidad de agua por su riqueza en recursos naturales que propician su producción. No obstante en algunas regiones del país existen problemas de disponibilidad de este recurso para consumo humano, y en varios casos se pueden identificar amenazas evidentes sobre las fuentes del mismo.

En particular en este artículo se trata el caso de Bogotá que debe atender con servicio de agua a una población de más de ocho millones de habitantes, que si bien cuentan actualmente con acceso al agua en condiciones relativamente buenas, también se pueden identificar amenazas sobre la posibilidad de conservar la calidad del servicio en el largo plazo.

Actualmente se encuentran en desarrollo diversos proyectos de inversión pública liderados principalmente por la empresa de Acueducto de Bogotá, cuya ejecución está orientada hacia el mejoramiento de la capacidad de abastecimiento del servicio de acueducto a la población del área de influencia de la empresa. Estos proyectos en desarrollo junto con otros que aún no arrancan, llenan en parte las necesidades de inversión que se requieren para garantizar el servicio de acueducto en un horizonte de mediano y largo plazo. Con este propósito es importante considerar los requerimientos del contexto territorial que circunda a la capital, teniendo en cuenta que de las condiciones del medio ambiente y de los recursos naturales que caracterizan estas zonas en cuanto a su conservación, es determinante para la regulación de la disponibilidad de agua para la capital.

En este trabajo se tuvo como objetivo destacar las condiciones generales en que se encuentran los sistemas de los cuales depende el abastecimiento de agua de Bogotá, para lo cual se adelantó una revisión bibliográfica sobre los temas propuestos, identificando de un lado el sistema medioambiental que soporta su abastecimiento, las debilidades que amenazan la garantía de continuidad de la disponibilidad de agua para la ciudad y la importancia económica que representaría disponer de un sistema complementario de abastecimiento.

\section{IMPORTANCIA DEL AGUA PARA CONSERVAR EL BIENESTAR Y EL NIVEL DE VIDA}

"La seguridad del agua es la disponibilidad de una cantidad y calidad aceptable del agua para la salud, medios de vida, los ecosistemas y la producción, junto con un nivel aceptable de riesgos relacionados con el agua para las personas, el medio ambiente, y las economías. " (Grey,D., and Sadoff,C., 2007, Pag. 547).

La disponibilidad de agua en las grandes, medianas y pequeñas ciudades, depende fundamentalmente de la capacidad de las zonas rurales para generarla, lo que posibilita a su vez obtener los servicios asociados a su disponibilidad. De la disponibilidad de agua en las ciudades depende en gran parte el nivel de vida de la población urbana y para ello, de acuerdo con los estándares internacionales de acceso al agua, este debe cumplir con criterios mínimos de regularidad, de calidad y precio (United Nations, s.f., pag.167).

Sin embargo la calidad del servicio de acueducto parece desmejorar en correlación inversa con el tamaño de la población. De acuerdo con encuesta realizada por iniciativa del BID en ciudades de América Latina en 2013, la población de las más grandes ciudades estudiadas son las que padecen más deficiencias en el servicio de agua, como es el caso de ciudad de México con $28 \%$ de insatisfacción, Sao Paulo con $8 \%$ y Buenos Aires con $7 \%$. Aunque Lima no está entre las ciudades más grandes dentro del estudio, también arrojó un nivel alto de insatisfacción con el $9 \%$. En el caso de Bogotá se obtuvo el 
menor nivel de insatisfacción con un 2\% (BID, 2013, pag. 10). ${ }^{1}$

Contar con suficiente agua en una gran ciudad no implica solamente el aseguramiento del líquido para los fines que normalmente se utiliza en los hogares. También es determinante para posibilitar actividades económicas que son esenciales para mantener el nivel de bienestar de la población; por ejemplo, la actividad industrial y comercial requiere servicio de agua eficiente y abundante; disponer de agua abundante conlleva la posibilidad de generar servicio de energía eléctrica, que mejora ostensiblemente el nivel de bienestar de familias rurales o urbanas. Que hay relación entre disponibilidad de agua y disponibilidad de energía eléctrica lo atestigua el grado de insatisfacción con el servicio de energía prestado en ciudades latinoamericanas donde también se alcanzan los mayores niveles en las ciudades más grandes como en el caso de México con $18 \%$, Sao Paulo $17 \%$ y Buenos Aires $18 \%$ (BID, 2013, pag.28). En las ciudades más pequeñas de la muestra se obtuvieron menores niveles de insatisfacción como es en Bogotá $4 \%$ de insatisfacción y en lima el 3\% (ibid).

La satisfacción de las necesidades por agua que requieren las ciudades modernas, implica administrar el recurso eficientemente y de manera sostenible, lo cual quiere decir que se debe ejercer gobernabilidad sobre el agua. Gobernabilidad del agua significa que las autoridades responsables posean la capacidad necesaria para generar las políticas adecuadas y la capacidad de ejecutarlas (Solanes M., y Jouravlev, A., 2005, pag. 8). Para lograr gobernabilidad es importante que todos los proyectos e inversiones relacionados con el agua garanticen, como mínimo, contar con estudios de factibilidad económica, alta productividad del agua implicada y eficiencia económica y entrópica en su uso. (ídem).

1 Población en 2013 en Ciudad de México (21.1 mills.), Sao Paulo (19.0 mills.), Bos. Aires (12.8 mills.), Bogotá (9,2 mills.) y Lima (8.4 mills.) en 2013, según el BID (2013)

\section{LAS FUENTES DE ABASTECIMIENTO DE AGUA EN LA CIUDAD DE BOGOTA}

De acuerdo con Mumford, (1991, citado por UN, s.f.) hace apenas algo más de cien años desde que se aceptó por parte de los países más desarrollados en Norteamérica y Europa que la disponibilidad permanente de servicio de acueducto en las ciudades, y la organización de la administración pública de estas para garantizar la seguridad del abastecimiento de agua, constituye un factor esencial para el bienestar de las poblaciones que habitan la ciudad. Sin embargo en 1976, en la Conferencia de Naciones Unidas sobre Asentamientos Humanos, hábitat, ( $y$ en sucesivas conferencias de la ONU) se reiteraba sobre esa necesidad, sin que a la fecha se cumplan los objetivos propuestos de disponer de servicio de agua segura y saneamiento adecuado universal.

El crecimiento de las ciudades da lugar a un problema de inmensa magnitud en lo que se refiere a lograr suficiente abastecimiento de agua dulce para atender las necesidades de su población. La paradoja de esta necesidad es que, según lo expone Naciones Unidas "---las ciudades requieren un enorme suministro de agua dulce y, a su vez, tienen un gran impacto sobre el agua dulce" (United Nations, 2015).

Las necesidades de agua están en función directa de la tasa de crecimiento de la población, que a su vez determina el crecimiento de actividades de producción industrial y agropecuarias, lo cual exige que la inversión de los gobiernos municipales y nacionales para mejorar la eficiencia en el aprovisionamiento y consumo de agua en las zonas urbanas sea una prioridad de las politicas públicas. Para lograr éxito en semejante desafío se requiere un gran esfuerzo financiero y de control en los usos que se hacen del recurso hídrico en las ciudades. En el VII foro mundial del agua, realizado en Daegu, Corea, entre el 12 y el 17 de abril de 2015, se hizo un pronóstico sobre las necesidades futuras de agua, energía y alimento preocupante. En 
LIINEA DE INVESTIGACIÓN: ECONOMIA SOCIAL Y DISTRIBUCIÓN DEL INGRESO Y LA RIQUEZA

efecto la ONU pronosticó que para el año 2030, se prevé un aumento en el mundo de la demanda de energía del $50 \%$, agua $40 \%$ y alimentos $35 \%$ (ONU-DAES, 2015).

En el caso de Bogotá, el futuro de la disponibilidad de agua depende del manejo que se dé a los recursos naturales existentes y que retroalimentan la capacidad de generar agua en la sabana de Bogotá y en los páramos y áreas de bosques y montañas que rodean a la ciudad.

El abastecimiento del servicio de agua potable de Bogotá está conformado básicamente por sistemas de embalses y páramos. Está el sistema de Chingaza con capacidad para abastecer el $\mathbf{8 0 \%}$ de las necesidades de agua de la capital, conformado por los embalses de Chuza y San Rafael, con capacidad de almacenamiento de 227 y 63.5 millones de metros cúbicos respectivamente. De otra parte está el sistema Sumapaz que suministra el $5 \%$, conformado por los embalses de Chisacá con capacidad de 7,4 millones de metros cúbicos y La Regadera que tiene una capacidad total de 3,8 millones de metros cúbicos. Además está el páramo de Guerrero que provee el $15 \%$ del agua que requiere la ciudad. (EAB, página digital, 2015)

Uno de los pulmones ambientales para generación de agua en reserva para la capital del país se encuentra al norte de la ciudad, localizado en la cuenca del río Bogotá donde se ha conformado un sistema de embalses o reservorios de agua, del cual hace parte el embalse de Tominé con capacidad para 658 mills. de metros cúbicos, que provee el agua de los municipios de Guatavita, Sesquilé y Guasca, y les genera la energía eléctrica. También está el embalse del Sisga con capacidad de 90,1 millones de metros cúbicos y el embalse del Neusa con capacidad de 144,5 millones de metros cúbicos. Actualmente el abastecimiento de agua para Bogotá, depende fundamentalmente de tres páramos como son Sumapaz, Guerrero y Chingaza, los tres pertenecientes a la vertiente de la Orinoquia.
La dependencia del servicio de acueducto en la zona urbana de la capital de Colombia con respecto al funcionamiento satisfactorio de los embalses y plantas de tratamiento del agua transportada a la ciudad, implica riesgos para la seguridad de abastecimiento asociados con diversas posibles causas. En general estas mismas causas afectan en mayor escala la seguridad de disponibilidad del recurso agua a la población mundial como un todo. De acuerdo con fuentes especializadas en el tema (InfoReSources Focus, 2003, No 1/03, Pag.3) el aumento del consumo de agua está poniendo en un alto riesgo la posibilidad de atender a las necesidades de la población mundial, que junto con otras causas como las siguientes, también constituyen amenazas para la disponibilidad futura de agua en Bogotá específicamente:

- Rápido crecimiento poblacional en las zonas urbanas.

- Se viene aumentando la proporción de agua utilizada para la industria, la agricultura y la ganadería, y reduciendo la proporción disponible para el consumo humano.

- Las zonas de páramo que abastecen el sistema de acueducto de Bogotá están siendo afectadas seriamente en su ecosistema por la deforestación y la ampliación de áreas destinadas a la ganadería y a la agricultura.

- Un alto porcentaje de aguas residuales se vierten en los ríos sin ningún tratamiento previo.

Todos los factores aquí señalados, afectan el valor entrópico del agua y la cantidad disponible, en particular para uso doméstico. "De a poco, a medida que la población y las actividades humanas se incrementan, los grandes cuerpos de agua, lagos, océanos y mares pasan a ser grandes cubetas de almacenammiento de aguas residuales." 
Gráfica 1. Reservorios de agua para generación de servicio de acueducto y energĺa eléctrica para Bogotá y municipios circunvecinos

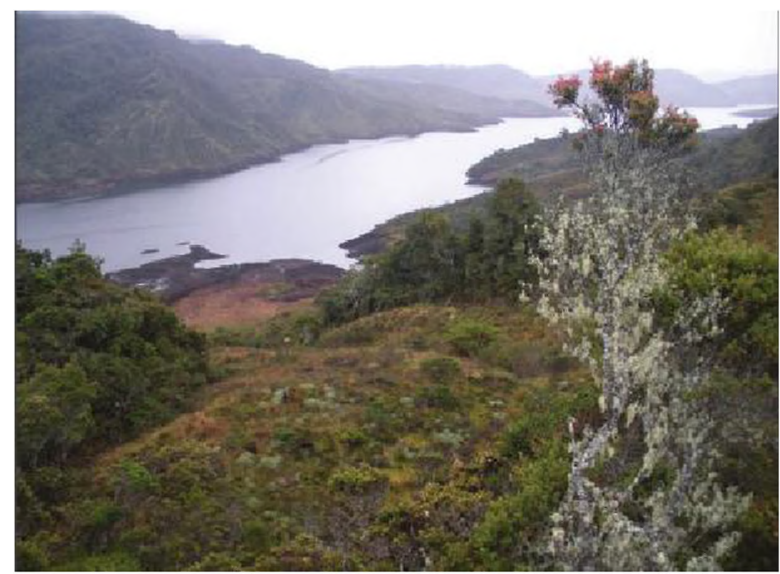

Embalse de Chuza, Parque Nacional de Chingaza.

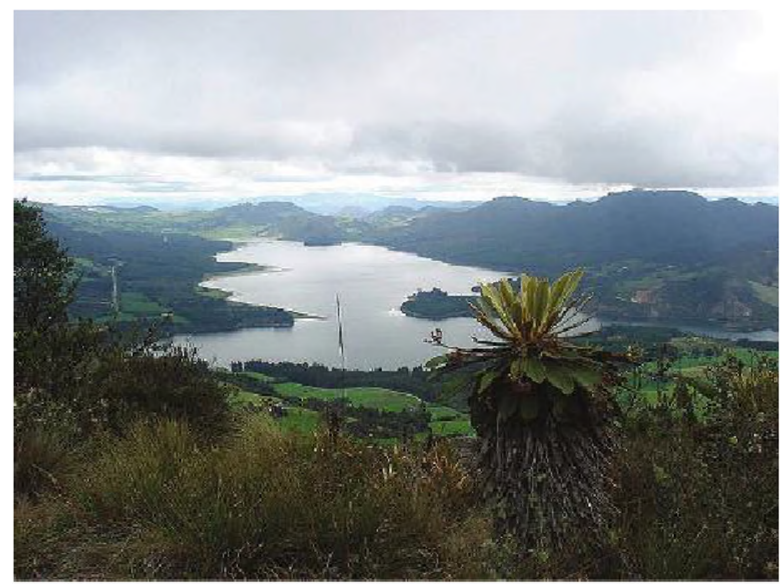

Páramo de Guerrero - Cundinamarca

Fuente: Google Maps, 2015

"Irresponsable e irreversiblemente, los seres humanos están transformando el mundo de las aguas naturales en un mundo de aguas residuales," (Antón, D. y otros, 2000, pag.373).

Evidentemente las causas arriba señaladas pueden tener los mismos impactos negativos, no sólo en Bogotá sino en Colombia, sobre la posibilidad de mantener la capacidad de atender las necesidades de agua a la población, lo cual requiere que se tomen medidas remediales para evitar que los factores señalados, entre otros, puedan precipitar

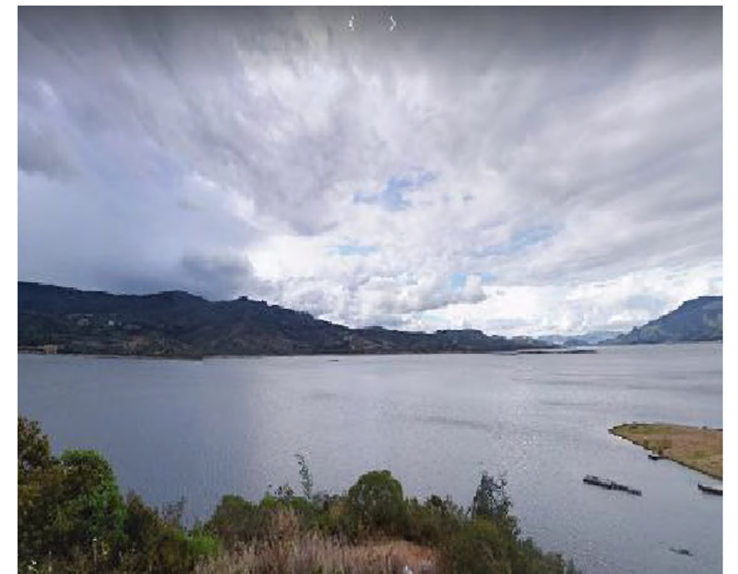

Embalse de Guatavita - Cundinamarca

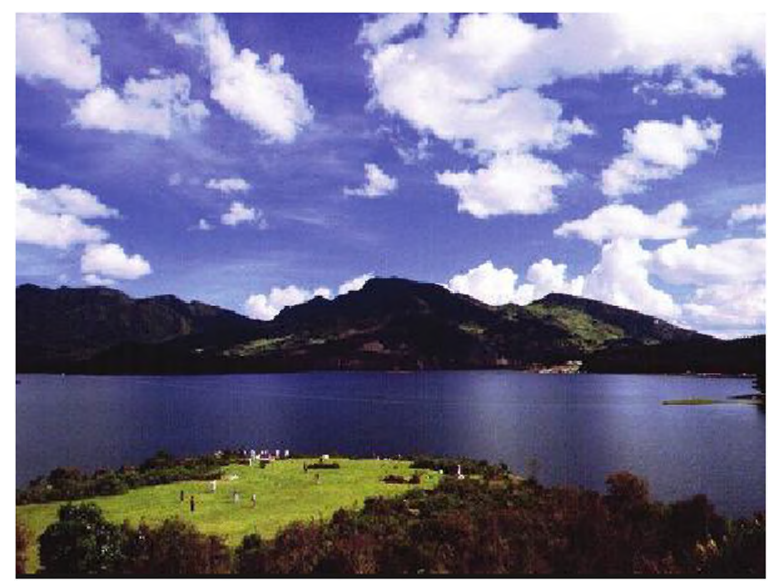

Represa del Neusa - Cundinamarca

graves problemas para el adecuado abastecimiento en el futuro.

\section{PROYECCION DE RIESGOS, NECESIDADES Y ABASTECIMIENTO DE AGUA PARA BOGOTA}

De acuerdo con Grey, D. y Sadoff, C.,(2006, Pag. 6.) "El desafío de crecimiento responsable es crecer incluyendo al mismo tiempo tanto la sostenibilidad ambiental como el desarrollo social. Un camino responsable es particularmen- 
LIINEA DE INVESTIGACIÓN: ECONOMIA SOCIAL Y DISTRIBUCIÓN DEL INGRESO Y LA RIQUEZA

te importante en el desarrollo del agua, ya que, dada la longevidad de la infraestructura de agua, muchas de las decisiones tendrán consecuencias a largo plazo. Además muchas de las decisiones, tanto las decisiones de actuar y no actuar puede tener consecuencias irreversibles".

Según las proyecciones realizadas en el Estudio Nacional del Agua - ENA, adelantado por el IDEAM (2010), para el año 2015 el número de cabeceras municipales con altos índices de escasez de agua llegaría a un número relativamente elevado. Para el caso de Bogotá, el Estudio del Agua estimó que podría tener un índice $D / O$ de escasez bajo, ubicado en un intervalo medio entre 11 y 20 en el caso extremo de año seco, (IDEAM, 2010, pag. 34).

Estudios más recientes sobre la disponibilidad de agua en la región Bogotá Cundinamarca, indican que las expectativas sobre la seguridad de abastecimiento del recurso agua para la región presentan dudas importantes, asociadas con los efectos que se podrían esperar por el cambio climático.. Es así como el Plan Regional Integral de Cambio Climático para la región PRICC, elaborado por un conjunto de entidades colombianas y extranjeras lideradas por el IDEAM y el PNUD comprometidas con la búsqueda de soluciones y estrategias para afrontar el cambio climático en la región, obtienen conclusiones preocupantes sobre el régimen dlimático de la región en el largo plazo. Algunas de las conclusiones del estudio indican que para los años entre 2041-2070 el régimen de lluvias se intensificará en un $10 \%$ a $30 \%$ en Bogotá y el centro del departamento de Cundinamarca, en tanto que se reducirían las lluvias en un $10 \%$ a $20 \%$ al oriente del departamento, con respecto al régimen actual y al occidente sobre el valle del Magdalena se esperaría una reducción de entre $20 \%$ a $40 \%$. (IDEAM, PNUD, 2014, pag.5). Las dos últimas conclusiones señaladas evidentemente afectan negativamente las expectativas de disposición de agua para la capital, en cuanto la reducción de lluvias recaen precisamente en las áreas que alimentan la oferta para la capital.
En el mismo trabajo citado se identificó el comportamiento probable de la disponibilidad hídrica para el año 2050 y se estableció que habría una "---mayor exposición por reducción en disponibilidad hídrica a lo largo de la Sabana de Bogotá, en municipios tales como Guachetá, Fúquene, Tausa, Facatativá, Subachoque, Cáqueza y Fómeque. En las zonas que alimentan el embalse de Chivor y Guavio, se prevén las más grandes caídas en disponibilidad". (IDEAM, PNUD, 2014, pag.5).

\section{ACCIONES PARA LA APLICACIÓN DE UNA POLÍTICA DE MANEJO DEL AGUA}

Los lineamientos de una política de manejo del recurso agua, de acuerdo a diferentes organizaciones de orden internacional, ONG y organismos gubernamentales coinciden en que debe ser acorde con la importancia estratégica que requiere la conservación de los recursos naturales y el medio ambiente, y en particular por el carácter esencial que el recurso agua representa para el bienestar de la población de cada país, región o ciudad. Con tal visión, la Declaracion de Dublin ${ }^{2}$ sobre el agua y el desarrollo sostenible emitió cuatro principios, "--- tendientes a reversar los efectos nocivos del abuso en el exceso de consumo, la polución y las crecientes amenazas de sequía e inundaciones".(The Dublin Statement, 1992). Los cuatro principios son los siguientes ${ }^{3}$.

Primer principio. El agua fresca es un recurso vulnerable y finito, esencial para el sostenimiento de la vida, el desarrollo y el medio ambiente.

En cuanto el agua sostiene la vida, se requiere un efectivo manejo holístico que inclu-

2 Esta declaración fue el resultado de una reunión de expertos sobre problemas relacionados con el agua llevada a cabo en The dublin statement on water and sustainable development, en Dublín entre el 26 y el 31 de enero de 1992.

3 Texto traducido del inglés por el autor. 
ya el desarrollo económico y social, junto con la protección de los ecosistemas naturales. Un efectivo manejo comprende la tierra y el agua utilizadas en todas las áreas que alimentan los acuíferos.

Segundo principio. El manejo y desarrollo del agua debe basarse en un enfoque participativo, incluyendo usuarios, planificadores y diseñadores de políticas a todos los niveles.

El enfoque participativo implica dar a conocer la importancia del agua entre los responsables políticos y el público en general. Esto significa que las decisiones se toman en el nivel más bajo posible, con una completa consulta pública y participación de los usuarios en la planificación y ejecución de proyectos de agua.

Tercer principio. Las mujeres juegan un papel fundamental en el abastecimiento, la gestión y protección del agua.

Este papel fundamental de las mujeres como proveedoras y usuarias del agua y guardianes del medio ambiente de la vida rara vez se ha visto reflejado en los arreglos institucionales para el desarrollo y gestión de los recursos hídricos. La aceptación e implementación de este principio requiere políticas positivas para hacer frente a las necesidades específicas de las mujeres y para equipar y capacitar a las mujeres a participar en todos los niveles en los programas de recursos hídricos, incluyendo la toma de decisiones y la aplicación, de manera que ellas determinen.

Cuarto Principio. El agua tiene un valor económico en todos sus usos competitivos $y$ debe ser reconocida como un bien económico.

Dentro de este principio, es vital reconocer primero el derecho básico de todos los seres humanos a tener acceso al agua potable y al saneamiento por un precio asequible. El fracaso en el pasado a reconocer el valor económico del agua ha llevado a usos despilfarradores y perjudiciales para el medio ambiente. La gestión del agua como un bien económico es una manera importante de lograr un uso eficiente y equitativo, y de fomentar la conservación y protección de los recursos hídricos.

Los cuatro principios enunciados en la declaración de Dublin han constituido guías importantes para oriemtar el manejo del recurso agua con responsabilidad en muchos países, y en particular para combatir los problemas más graves causados por la escasez de agua como son alivio de la pobreza, las enfermedades y la protección contra los desastres naturales,

Los principios enunciados se inspiran en la visión de la acción a desarrollar desde el concepto de Manejo Integrado del Recurso Agua (IWRM - Integrated Water Resource Management) concepto cuya formulación, además de la declaración de Dublín, se ha realizado en varias oportunidades como en la Tennessee Valley Authority en 1930, y en la Conferencia de las Naciones Unidas en Mar del plata en 1977. (Butterworth, J. y otros, 2010, pag. 69).

Otros autores estudiosos de las políticas en manejo del "problema del agua" coinciden en considerar que el IWRM es una manera útil y eficiente para abordar políticas de recuperación de la seguridad en la disponibilidad de agua para la sociedad actual. Al respecto Batchelor, Ch. (2007) considera que es una opción importante para el manejo de acciones que buscan la recuperación del recurso, porque se basa en el trabajo de las comunidades con base en la la complementación y coordinación de los diferentes estamentos de la sociedad en lugar de trabajar con base en la unidad de la microcuenca con comunidades muy específicas y limitadas en cuanto su visión del problema.

La reiterada renovación de la confianza intitucional mostrada en las propuestas y conceptos sobre el IWRM indica que es un modelo de trabajo por el agua que tiene virtudes valiosas para emprender actividades importantes en favor de este recurso. De ello se desprende que en el caso colombiano es un arquetipo de trabajo que se puede seguir. 
LIINEA DE INVESTIGACIÓN: ECONOMIA SOCIAL Y DISTRIBUCIÓN DEL INGRESO Y LA RIQUEZA

\section{VENTAJAS DE UN SISTEMA ALTERNATIVO DE ABASTECIMIENTO DE AGUA EN LA CIUDAD}

Otro punto de vista respecto al abastecimiento de agua para Bogotá hace referencia, no ya a las fuentes y métodos de abastecimiento de agua convencionales atrás señaladas, sino al que tiene que ver con el aprovechamiento del agua lluvia que se precipita sobre la ciudad y su área de influencia cercana.

En el cuadro 1 se presenta una clasificación que muestra los limitantes que afectan el uso del agua, dependiendo de la procedencia de la misma, los organismos vivos que contenga y los vertimentos de agua servida que la contaminen. El mayor nivel entrópico lo muestra el agua condensada en las nubes porque no está expuesta a contaminación aunque su valor se hace efectivo solamente cuando se convierte en precipitación de agua lluvia, de granizo o de rocío. El agua con mayor oportunidad de uso es la de lluvia, siendo la única en su forma natural con posibilidad de utilizarse como agua potable para consumo humano.

El agua lluvia se puede aprovechar en las áreas urbanas para satisfacer diversas necesidades de los hogares, y en actividades productivas que requieren agua en la ciudad, lo que de posibilitarse se dispondría de una fuente adicional del líquido que puede tener efectos muy positivos en diversos aspectos relacionados con su consumo. En este sentido se dependería menos de la capacidad de los embalses para el suministro corriente del servicio de acueducto; se reducirian las inversiones públicas requeridas para sostener la capacidad de abastecimiento de agua a la creciente población urbana; los costos del servicio de acueducto se disminuirían a los usuarios por la disminución del consumo de agua tarifada provista por la entidad responsable, entre otros beneficios.

Las condiciones básicas para lograr el aprovechamiento de las aguas lluvias que caen en el casco urbano de Bogotá y sus alrededores, implican un nivel de precipitaciones suficientes para sostener un promedio histórico de las mismas, lo que requiere desarrollar un programa exigente de conservación y recuperación de las coberturas bióticas forestales que rodean la capital, en particular los páramos, y la sabana de Bogotá. Además es necesario que el gobierno nacional y los gobiernos municipales de la región se propongan revertir, o por lo menos neutralizar las causas que propician el calentamiento global y la distorsión del régimen de lluviosidad.

La ventaja más evidente del aprovechamiento del agua lluvia por los usuarios del sistema de recolección, sería el ahorro en los costos de la factura por consumo de agua tarifada. Este ahorro en términos de volumen de agua ahorrada está determinado por el cociente entre la capacidad de acumulación-uso de agua Iluvia y el consumo total. (Palacio,N., 2010, pag.19). Aunque la relación entre las variables señaladas depende en parte del uso que se de al agua lluvia recuperada, porque el volumen puede ser mayor si se utiliza inclusive para consumo humano, lo cual no es descartable considerando que algunos estudios demuestran que ello es posible sin correr riesgo la salud. Es así como los investigadores Sazakli, E., y otros (2007) en un lapso de tres años recolectaron 300 muestras de agua, 156 de los tanques de almacenamiento que recibían directamente el agua lluvia, y las 144 restantes se extrajeron de aguas subterráneas de la región estudiada y llegaron a la conclusión de que las primeras eran más aptas para ser utilizadas como agua potable. (Citado por Palacio, N., op.cit).

La utilización del agua lluvia como sistema subsidiario de otras fuentes, representaría para el caso de Bogotá un margen importante de alivio al costo de los usuarios de todas las actividades económicas que podrían utilizar tal recurso, lo que incidiría en reducción de costos de operación para la industria y el comercio, costos de servicios públicos para las familias y para las instituciones gubernamentales. 


\begin{tabular}{|c|c|c|c|c|c|c|}
\hline \multirow{2}{*}{$\begin{array}{c}\text { Nivel } \\
\text { entropico }\end{array}$} & \multicolumn{2}{|c|}{ Aguas naturales } & \multirow{2}{*}{$\begin{array}{l}\text { Utilización } \\
\text { del agua } \\
\text { natural }\end{array}$} & \multirow{2}{*}{$\begin{array}{l}\text { Aguas residuales o } \\
\text { contaminadas }\end{array}$} & \multirow[b]{2}{*}{ Posición geológica } & \multirow[b]{2}{*}{ Presencia de vida } \\
\hline & $\begin{array}{l}\text { Aguas superficiales, } \\
\text { atmosféricas }\end{array}$ & Aguas subterréneas & & & & \\
\hline 10 & $\begin{array}{l}\text { Nubes altas, recién } \\
\text { condensadas }\end{array}$ & & \begin{tabular}{|l|} 
Agua desti- \\
lada \\
\end{tabular} & & Atmostérica, elevada & Organismos muy escasos, pocos nutrientes \\
\hline \begin{tabular}{|l|}
9 \\
8
\end{tabular} & $\begin{array}{l}\text { Nubes bajas, lluvia, } \\
\text { nieve }\end{array}$ & & Agua potable & & Atmosférica, baja & Organismos escasos, pocos nutrientes \\
\hline 7 & \begin{tabular}{|l|} 
Manantiales, torrentes \\
de montañas
\end{tabular} & & \begin{tabular}{|l|}
$\begin{array}{l}\text { Aguas terma- } \\
\text { les }\end{array}$ \\
\end{tabular} & & Cimas, cabeceras valles & $\begin{array}{l}\text { Organismos de abundancia escasa a inter- } \\
\text { media }\end{array}$ \\
\hline 5 & \begin{tabular}{|l|} 
Cursos medios de \\
ríos, lagos medios, \\
emisarios de ciertos \\
humedales \\
\end{tabular} & $\begin{array}{l}\text { Napas hipodérmicas, } \\
\text { acuíferos poco profun- } \\
\text { dos no contaminados }\end{array}$ & $\begin{array}{l}\text { Aguas para } \\
\text { riego }\end{array}$ & Lluvia muy ácida & $\begin{array}{l}\text { Zonas de colinas, sierras } \\
\text { bajas, subsuelo de poca } \\
\text { profundidad }\end{array}$ & Organismos abundantes \\
\hline 4 & $\begin{array}{l}\text { Cursos bajos de río, } \\
\text { lagos de llanura, hu- } \\
\text { medales oxigenados }\end{array}$ & \begin{tabular}{|l} 
Agua subterránea \\
profunda dulce. Poco \\
profunda ligeramente \\
salobre.
\end{tabular} & $\begin{array}{l}\text { Aguas para } \\
\text { riego }\end{array}$ & $\begin{array}{l}\text { Drenajes de riego, } \\
\text { agua residual tratada }\end{array}$ & $\begin{array}{l}\text { Llanuras, colinas bajas } \\
\text { subsuelo medianamente } \\
\text { a muy profundo. }\end{array}$ & $\begin{array}{l}\text { Organismos muy abundantes en ríos y lagos, } \\
\text { localmente exceso de nutrientes. Vertidos de } \\
\text { aguas de riego pueden provocar procesos de } \\
\text { eutroficación. }\end{array}$ \\
\hline 0 & Mares y lagos salados & $\begin{array}{l}\text { Agua subterránea } \\
\text { salada. }\end{array}$ & \begin{tabular}{|l|} 
Aguas balnea- \\
rias
\end{tabular} & $\begin{array}{l}\text { Vertidos urbanos e } \\
\text { industriales medios }\end{array}$ & $\begin{array}{l}\text { Nivel del mar, zonas } \\
\text { continentales depri- } \\
\text { midas, subsuelo de } \\
\text { profundidad variable }\end{array}$ & $\begin{array}{l}\text { Organismos muy abundantes en mares y } \\
\text { lagos, escasos en vertidos urbanos. } \\
\text { Los vertidos urbanos provocan frecuentes } \\
\text { procesos de eutroficación. }\end{array}$ \\
\hline $0 a-5$ & Salmueras & Salmueras subterráneas & $\begin{array}{l}\text { Producción } \\
\text { de sal }\end{array}$ & \begin{tabular}{|l|} 
Vertidos urbanos e \\
industriales altamen- \\
te contaminados \\
\end{tabular} & Salmueras subterráneas & $\begin{array}{l}\text { Escasos organismos debido a la toxicidad, } \\
\text { procesos de eutroficación posibles localmente }\end{array}$ \\
\hline$<-5$ & Salinas & $\begin{array}{l}\text { Yacimientos de } \\
\text { sal }\end{array}$ & \begin{tabular}{|l|}
$\begin{array}{l}\text { Producción de } \\
\text { sal industrial }\end{array}$ \\
\end{tabular} & \begin{tabular}{|l|} 
Vertidos industriales \\
de alta toxicidad
\end{tabular} & Yacimiento de sal & Ausencia de organismos \\
\hline
\end{tabular}

Fuente: Antón, D. y otros. (2000). El valor del agua. Ecosistemas humanos y biodiversidad. Centro Interamericano de Recursos del Agua, Universidad Autónoma del Estado de México, Toluca. 
LIINEA DE INVESTIGACIÓN: ECONOMIA SOCIAL Y DISTRIBUCIÓN DEL INGRESO Y LA RIQUEZA

El régimen de lluvias en Bogotá permite el aprovechamiento eficiente del sistema de aguas lluvias, como lo indica su aplicación en algunos casos en la ciudad. Por ejemplo según Ballén, Galarza y Ortiz, (2.006) el local de Alkosto del barrio Venecia que cuenta con una cubierta de aproximadamente 6.000 metros cuadrados que captan alrededor de 4.820 metros cúbicos de agua lluvia anualmente, representa el $75 \%$ del consumo de agua del almacén. (Citado por Palacio, pag. 17).

El recurso de recolección de agua de lluvia podría ser una solución a la sostenibilidad del servicio de acueducto en Bogotá en el largo plazo si se incluye de manera amplia en el sistema de gestión del agua urbana de la ciudad, ya que mitigaría los problemas de disponibilidad de agua que se puede esperar por efecto del cambio climático, como afirman documentos de la International Institute for enviroment and development - iied, (Batchelor, 2007), que se presentan en forma de largas épocas de lluvias (fenómeno de la Niña) y de épocas de sequías (fenómeno del niño), lo que genera escasez de agua para atender las necesidades de la población o problemas de inundaciones, lo que atenta contra la salud de millones de personas. También se reduciría la presión sobre las fuentes tradicionales de agua teniendo en cuenta que el rápido crecimiento de la población en Bogotá causa una gran presión sobre la capacidad de prestación de servicio de acueducto en la ciudad y sus alrededores, lo que llevará a que la demanda supere cada vez más a la capacidad de oferta, y contribuiría a evitar las inundaciones. (Sadia, R.,2014).

Un importante efecto que se desprende del aprovechamiento del agua lluvia es la reducción de la pobreza, y el mejoramiento de las condiciones de vida de las familias pobres que utilicen ese recurso. Debe considerarse que el agua más cara del país es la de Bogotá, que según la Comisión de Regulación de Agua -CRA- en mayo de 2015 estaba costando $\$ 2.210$ pesos el metro cúbico, lo que dificulta el acceso al agua de la población más pobre.

\section{CONCLUSIONES}

La disponibilidad de agua en los entornos donde habitan las poblaciones urbanas y rurales es un factor determinante para mantener un nivel de bienestar aceptable para los seres humanos que las habitan y para desarrollar eficientemente las actividades económicas que allí se concentran.

De la disponibilidad de agua dependen también en gran parte las posibilidades de generar otros bienes sociales necesarios para el bienestar, como por ejemplo la energía eléctrica.

La seguridad de disponibilidad de agua en Bogotá se encuentra amenazada particularmente, porque la cobertura vegetal propia del ecosistema de los páramos que la bastecen de agua se viene afectando seriamente, debido a la deforestación causada por ganaderos y agricultores allí afincados. Además el cambio climático afectaría negativamente el régimen de lluvias en la zona del norte, donde se ubican importantes reservorios de agua para la capital.

Para garantizar la disponibilidad de agua se requiere que se administre este recurso eficientemente, es decir que exista gobernabilidad del agua, que significa que las autoridades responsables posean la capacidad necesaria para generar las políticas adecuadas y la capacidad de ejecutarlas.

Los principios enunciados de acuerdo con el concepto del Manejo Integrado del Recurso Agua ( IWRM - Integrated Water Resource Management) contituyen un importante referente para orientar la política de manejo de agua para Bogotá y para el país.

El aseguramiento del agua de Bogotá en el futuro se puede afianzar introduciendo sistemáticamente el aprovechamiento del agua Iluvia para todas las acividades económicas y sociales que requieren agua, lo cual se posibilita por el régimen de lluvias propio de la ciudad. De acometerse esta iniciativa se obtendrían importantes beneficios en reducción de la pobreza, reducción de costos de producción empresariales y de los hogares por concepto de servicio de acueducto. 


\section{REFERENCIAS}

Antón, D. y otros. (2000). El valor del agua. Ecosistemas humanos y biodiversidad. Centro Interamericano de Recursos del Agua, Toluca, Universidad Autónoma del Estado de México.

Antón, D. y otros, ( 2000 ). Sequía en un mundo de agua. Montevideo, Ur., Toluca, Mex.; Piriguazú Ediciones, UAEM - Centro Interamericano de recursos del agua, Univ. Autónoma del Estado de México.

Ballén, J.A., Galarza, M.A., y Ortiz M., (2006). Sistemas de Aprovechamiento de Agua Lluvia para Vivienda Urbana. VI SEREA - Seminário lberoamericano sobre Sistemas de Abastecimento Urbano de Água., (2006)

Batchelor, Ch.(2007). Water governance literature assessment, International Institute for Environment and Development. London WC1X $8 \mathrm{NH}$, UK.

BID (2013). Megaciudades e infraestructura en América Latina. Lo que piensa su gente. Recuperado de : BIDinfraestructura@iadb.org

Butterworth, J.; Warner, J.; y otros.(2010). Finding practical approaches to Integrated Water Resources Management; Water Alternatives 3(1): 68-81.

Diaz Delgado et. Al, (2003). Metodología entrópica para la gestión integrada de cuencas hidrológicas; Centro Interamericano de Recursos del Agua, Cira-UAEM México.

Empresa de Acueducto de Bogotá.(2015). Recuperado de: http://www.acueducto.com.co/

EAAB. Proyectos de inversión. (2015) Camino a la excelencia ambiental. Recuperado en: http://www.acueducto.com.co/wpsv61/wps/ portal/!ut/p/c5/04_SB8K8xL L M 9 M S Z P y 8 x B z 9 C P0os 3gLw2DfYHMPIwN cyMXA09HV1cLM2MTJ5MgE6B8pFm8s7ujh4m5j4GBv1GYgYGRn2IwoEFosLGBpzEB3eEg-_DrB8kb4ACOBvp-Hvm5qfqR-IHmOOwJcg0004_MSU1PTK7UL8iNMMgyCVUEAFxrP4E!/ dl3/d3/LOIJSkIna2shLOICakFBTXIBQkVSQOIBISEvWUZOQzFOS18yN3chLzdfODFTT VM3SDIwTzcyRDBJQUVF ODYZNFJFUTY!/?WCM_PO RTLET=PC_7_81SMS 7 H2 0O72DOIAEE $\overline{8} 634$ REQ6 WCM\&WCM_GLOBAL_CONTEXT=/wps/wcm/connect/ eaabv6/sacueducto/aambiental/aambsecprincipal/aambientalhumedales

Grey D. y Sadoff, C. (2007). ¿Hundirse o Nadar? La seguridad hídrica para el crecimiento y el desarrollo. Política Hídrica 9: 545-557.

Grey, D. and Sadoff, C., (2006). Water for Growth and Development- A Theme Document of the 4th World Water Forum; Comisión Nacional del Agua; Mexico City.
IDEAM . (2010). Estu dio Nacional del Agua. Instituto de Hidrología, Meteorología. $y$ Estudios Ambientales. Bogotá D.C. recuperado de: https://www.siac. gov.co/contenido/contenido. aspx?cat ID $=874 \&$ con ID $=910$

InfoReSources Focus, (2003). No 1/03. Recuperado de: www.inforesources.ch

Movimiento Agua y Juventud y Federación Argentina de Municipios (FAM). (2015). Seminario Agua para Nuestro Futuro: Camino al $7^{\circ}$ Foro Mundial del Agua, Argentina.

Mumford, L. (1991). The City in History. Londres, Penguin Books. RICS International Paper Series, Royal Institution of Chartered

ONU-DAES.(2015). Decenio internacional para la acción - El agua fuente de vida" 20052015. Recuperado en: http:// waterforlifeconf2015.org/eng/

PALACIO, N., (2010). Propuesta de un sistema de aprovechamiento de agua lluvia, como alternativa para el ahorro de agua potable, en la institución educativa María Auxiliadora de caldas, Antioquia; Trabajo de grado, Universidad de Antioquia, Medellín. Reforestación zonas naturales. Recuperado en: http:// www.revistavirtualpro.com/revista/reforestacion-y-restauracionde-zonas-naturales/19

Sadia R. y otros. (2014). Sustainability of Rainwater 
LINNEA DE INVESTIGACIÓN: ECONOMIA SOCIAL Y DISTRIBUCIÓN DEL INGRESO Y LA RIQUEZA

Harvesting System in terms of Water Quality, The Scientific World JournalVolume 2014, article ID 721357, 10 pages.

Sazakli, E., Alexopoulos, A., y Leotsinidis, M.(2007). Rainwater harvesting, quality assessment and utilization in Kefalonia Island, Greece. Water research 41, 9 (2007), 2039-47.

Solanes M., y Jouravlev, A., (2005).Integrando economía, legislación y administración en la gestión del agua y sus servicios en América Latina, Santiago de Chile, CEPAL, serie Recursos naturales e infraestructura No. 101,

The Dublin statement on water and sustainable development (1992) Recuperado de:

http://www.wmo.int/pages/ prog/hwrp/documents/english/ icwedece.htm|\#p1
United Nations, UN water. (2015) “International Decade for Action. Water for life 20052015". Recuperado de: http:// www.un.org/waterforlifedecade/water_cities.shtml.

Veenhuisen, R. and Prieto C., M. Manual de Captación y Aprovechamiento del Agua de Lluvia. Experiencias en América Latina. Serie: Zonas Áridas y Semiáridas $\mathrm{N}^{\circ} 13.1991,1-5$. 